\title{
REVIEW
}

\section{Surgery of Intracranial Aneurysms at Yonsei University: 780 Cases}

\author{
Kyu Chang Lee \\ Department of Neurosurgery, Yonsei University College of Medicine, Seoul, Korea
}

(Received for publication on September 28, 1990)

\begin{abstract}
Seven hundred and eighty patients with intracranial aneurysm, which were surgically treated by the author since 1976, were analyzed. Strategies important for intracranial aneurysm surgery were the timing of surgery, preoperative preparation and intraoperative management. The best management outcome could be achieved by early operation, removal of subarachnoid blood clot, maintenance of circulating blood volume, administration of nimodipine, and meticulous surgical tactics to avoid pitfalls. Indications for aneurysm surgery in the acute phase were determined by intracerebral hematoma, angiographic findings, clinical grade, general physical status and readiness of the surgical team. Important goals to be considered during the operation were obtaining a slack brain, preparation of proximal control, protection of the brain, awareness of microsurgical anatomy, and complete dissection of the sac. The morbidity and mortality were $2.7 \%$ and $4.0 \%$, respectively. The mortality was attributed to intracranial causes in 20 cases (poor grade, delayed ischemic deficits, rebleeding, postoperative infarction, and postoperative epidural hematoma), extracranial causes in 7 cases (pulmonary embolism, heparin induced intracerebral hemorrhage, hepatic failure, myocardial infarction, and gastrointestinal bleeding), and unknown problems in 5 cases. The postoperative intracerebral hemorrhage occurred in 16 cases and seemed to be caused by one or more of the following events: cerebral infarction developed during the preoperative period, occlusion of the cerebral veins during the Sylvian dissection, cerebral retraction and/ or sudden change of intracranial hemodynamics. Hydrocephalus, almost always a communicating type as confirmed by isotope cisternography, was managed by lumboperitoneal shunt. It is concluded that crucial factors in obtaining the best overall management outcome of the ruptured intracranial aneurysms are early referral, proper preoperative evaluation, meticulous planning of the surgery including the time of surgery, and finally, alert team work. (Keio J Med 40 (1): 1-5, March 1991)
\end{abstract}

Key words: tactics, timing of surgery, outcome

Seven hundred and eighty patients with intracranial aneurysm, which were treated surgically by author since 1976 at the Yonsei University Hospital, were analyzed. Out of the 780 patients (745 ruptured, 35 unruptured), 728 patients $(93.3 \%)$ showed favorable outcome, while 52 patients $(6.7 \%)$ had unfavourable outcome including $4.0 \%$ mortality (Table 1 ).

\section{Timing of Surgery Versus Outcome}

During the last six years (from Jan. 1, 1984 to Mar. $16,1990), 30.4 \%$ (136 patients among 448 ruptured aneurysms) were treated surgically within rupture day three.
Strategies important for successful intracranial aneurysm surgery include: obtaining slack brain, security of proximal control (temporary clipping), protection of the brain, awareness of microsurgical anatomy especially the perforating branches, complete dissection of an aneurysmal sac and proper selection of a clip. Indications for aneurysm surgery in the acute phase were determined by intracerebral hematoma requiring urgent decompression, definitive angiographic finding demonstrating saccular aneurysm, location and size of the aneurysm (giant aneurysms anticipating prolonged brain retraction and aneurysms of the posterior circulation were excluded), clinical grade (Hunt and Hess's grade 1 to 3 , under 65 year-old without significant systemic diseases

Reprint requests to: Dr Kyu Chang Lee, Department of Neurosurgery, Yonsei University College of Medicine, CPO 8044, Seoul, 120-752 Korea 
Table 1 Results of Operative Treatment.

\begin{tabular}{lccc}
\hline Outcome & Ruptured & Unruptured & Total \\
\hline Good & 626 & 32 & $658(84.3 \%)$ \\
Fair & 68 & 2 & $70(9.0 \%)$ \\
Poor & 21 & 1 & $21(2.7 \%)$ \\
Dead & 30 & $31(4.0 \%)$ \\
\hline Total & 745 & 35 & $780(100.0 \%)$ \\
\hline
\end{tabular}

were included), and, finally, readiness of the surgical team.

\section{Emergency surgery}

Thirty patients were operated within $24 \mathrm{hrs}$ of admission because of intracerebral hematoma, mostly due to ruptured aneurysm of the middle cerebral artery. Fourteen cases $(47.7 \%)$ showed favorable outcome, while 16 cases $(53.3 \%)$ had unfavourable results.

\section{Elective early surgery}

One hundred thirty-one patients were subjected to elective early surgery within rupture day three. Among them, 125 patients $(95.4 \%)$ showed favorable outcome and 6 cases $(4.6 \%)$ showed unfavorable results.

\section{Delayed surgery}

Five hundred and thirty-one patients were operated on rupture day four or later. Among them 551 patients $(94.8 \%)$ showed favorable outcome and 29 patients $(5.2 \%)$ showed unfavorable outcome (Table 2 ).

We were very careful in starting early surgery. A few patients with good surgical risk were subjected to early surgery since 1983 . Thereafter, we were convinced that the surgery in the acute phase was feasible in spite of "angry brain" that was frequently encountered. The operation in the acute phase was facilitated by adequate drainage of the cerebrospinal fluid through an spinal catheter which was inserted after anesthetic induction.

Table 2 Timing of Surgery Versus Outcome.

\begin{tabular}{lrrrrr}
\hline \hline & \multicolumn{2}{c}{ Within 3 Days } & & \multicolumn{2}{c}{ On Day 4 or Later } \\
\cline { 2 - 3 } & Emergency & \multicolumn{1}{c}{ Early } & & Delayed & Total \\
\hline No. of pts. & 30 & 131 & & 580 & 741 \\
Good & $9(31.0)$ & $112(85.5)$ & & $504(86.7)$ & $625(84.2)$ \\
Fair & $5(16.7)$ & $13(9.9)$ & & $47(8.1)$ & $65(8.8)$ \\
Poor & $6(20.0)$ & $1(0.8)$ & & $13(2.4)$ & $20(2.8)$ \\
Dead & $10(33.3)$ & $5(2.8)$ & & $16(2.8)$ & $31(2.4)$ \\
\hline
\end{tabular}

\section{Surgical Mortality}

Thirty-two (4.0\%) out of the 780 patients died of various intracranial (20 patients) and extracranial (7 patients) causes after surgery. Seven deaths were attributed to the initial insult of subarachnoid hemorrhage, six death due to delayed cerebral ischemia, rebleeding in four cases, cerebral infarction in two cases and one case was complicated by an epidural hematoma. Extracranial causes of surgical mortality were pulmonary embolism in three cases; the other patients had heparin induced intracerebral hemorrhage, hepatic failure, myocardiac infarction and upper gastrointestinal bleeding. Five patients died of unknown causes (Table 3).

Table 3 Cause of Death (32 patients).

\begin{tabular}{|c|c|c|c|c|}
\hline $\begin{array}{l}\text { Intracranial } \\
\text { ( } 20 \text { cases) }\end{array}$ & & $\begin{array}{c}\text { Extracranial } \\
\text { (7 cases) }\end{array}$ & & $\begin{array}{l}\text { Unknown } \\
\text { (5 cases) }\end{array}$ \\
\hline $\begin{array}{l}\text { initial insult } \\
\text { delayed cerebral } \\
\text { ischemia }\end{array}$ & 7 & $\begin{array}{l}\text { pulmonary embolism } \\
\text { heparin induced intra- } \\
\text { cerebral hemorrhage }\end{array}$ & 3 & \\
\hline $\begin{array}{l}\text { rebleeding } \\
\text { cerebral }\end{array}$ & 4 & hepatic failure & 1 & \\
\hline $\begin{array}{l}\text { infarction } \\
\text { epidural }\end{array}$ & 2 & myocardiac infarction & 1 & \\
\hline hematoma & 1 & upper GI bleeding & 1 & \\
\hline
\end{tabular}

\section{Delayed Cerebral Ischemia and Calcium Antagonist}

The effect of the calcium antagonist nimodipine on the feline cerebral cortical blood flow in experimentally induced subarachnoid hemorrhage was studied. Subarachnoid hemorrhage was induced in 25 cats by a slow injection of fresh autologous arterial blood into the cisterna magna. The calcium antagonist nimodipine increased cerebral cortical blood flow significantly in experimentally induced subarachnoid hemorrhage, without considerable change in systemic mean arterial blood pressure. Promising results were obtained in the trials in the treatment and prevention of cerebral ischemia caused by vasospasm following subarachnoid hemorrhage. ${ }^{1}$

Forty-three patients with subarachnoid hemorrhage entered a nimodipine trial to determine the efficacy of the durg in preventing vasospasm and to evaluate the tolerance of this calcium channel blocker. Thirty-three patients completed the study. Treatment was started within four days of initial bleeding and continued for two weeks. Delayed neurological deficits developed in seven of the 33 patients; four from vasospasm, two from elevated intracranial pressure, and one from recurrent bleeding. The incidence of symptomatic vasospasm which developed after nimodipine treatment was $12.1 \%$, about one third of the rate experienced at the same institute 
during the five years $(32.2 \%)$. Twenty-five patients were operated on without surgical mortality, and the morbidity rate was $8 \%$. Side effects due to nimodipine treatment were reversible and insignificant. This study suggests that treatment with a calcium channel blocker that has a selective cerebrovascular effect may prevent or reduce the incidence of delayed ischemic deficits in patients with aneurysmal subarachnoid hemorrhage. ${ }^{2}$

\section{Postoperative Intracerebral Hemorrhage}

The author encountered 16 cases who were complicated by intracerebral hemorrhage (ICH) following aneurysm surgery. Hemorrhage was found in the area of infarction in 7 cases. The veins were injured in the Sylvian fissure in 8 cases. In 7 cases, the cortex was contused by retraction. Extensive dissection to expose the aneurysm was responsible in 2 cases, and no significant cause was identifiable in one case. Hematomas were found in the cortex, deep white mater or the caudate. In two cases, the hemorrhage occurred in the hemisphere contralateral to the surgical procedure. Deliberated arterial hypotension was induced in 7 cases, and temporary clipping of the parent artery was done in 8 cases. There was no premature rupture. In four cases the hematoma had to be evacuated. The average 14-month follow-up examination revealed no neurological deficit related, to the $\mathrm{ICH}$. It is concluded that, in order to prevent this complication, one has to check the immediate preoperative CT scan to detect cerebral infarction, prevent sudden fluctuation of arterial blood pressure during both the intraoperative and immediate postoperative period, avoid injury of the cerebral veins and, finally, give great care to cerebral retraction. ${ }^{3}$

\section{Hydrocephalus}

The incidence of hydrocephalus, ventricular dilatation on brain CT scan, among the patients who were operated on during the period from 1987 to 1989 , was 80 out of 223 patients. Shunt surgery was necessary in 25 patients $(11.2 \%)$. For the symptomatic hydrocephalus, radioactive isotope cisternography was performed to confirm communicating hydrocephalus and to determine the need for lumboperitoneal shunt surgery. Lumboperitoneal shunt was thought to be a very simple extracranial procedure which reduces the complications from ventriculoperitoneal shunt such as subdural fluid collection, subdural hematoma, slit ventricle syndrome and conversion from communicating hydrocephalus to noncommunicating hydrocephalus. Opening of the laminar terminalis and/or Liliequist membrane in a case with hydrocephalus was useful to obtain a slack brain; however, it was not clear whether this procedure obviated the shunting incidence. ${ }^{4}$

\section{Tactics to Avoid Pitfalls}

\section{Meticulous review of cerebral angiogram before surgery to detect an associated microaneurysm}

A tiny protrusion of the proximal medial wall of the internal carotid artery was noticed in a patient with ruptured aneurysm in the anterior communicating artery. This microaneurysm was confirmed during the surgery. Since these incidental lesions are thin-walled, if not anticipated it, the lesion could inadvertently rupture during pterional approach. Such a lesion was found on proximal portion of the internal carotid artery or bifurcation of the internal carotid artery. Sometimes these microaneurysms or preaneurysmal lesions were not detected on angiogram and found only during surgery.

\section{Partially thrombosed aneurysm}

Thrombosed aneurysms should be anticipated through review of CT scan or MRI, since the management of these lesion demands specific attention during dissection and clipping. Manipulation of a thrombosed aneurysm may result in embolization. Clips may not be closed completely or may slip down toward the parent artery compromising its patency.

\section{Aneurysm of the anterior communicating artery}

It is not uncommon to find an aneurysm arising at the end of the left A1 facing right and atresic right A1. The left pterional approach would be appropriate to secure proximal control of major afferent artery, left A1. However, one can avoid manipulating the dominant hemisphere (left) by choosing the right approach. It is possible to expose the left A1 through right pterional approach. This procedure provides better visualization of both sides of the aneurysmal neck and a safer clip application compared to the left approach. However, one has to be aware of the location of the aneurysm and has to avoid premature rupture before access to the left A1. In interhemispheric type or posteriorly directed anterior communicating artery aneurysm, complete dissection of posterior aspect of the both A2 and anterior communicating artery and preservation of hypothalamic perforators are mandatory. Prechiasmatic type or anteriorly directed anterior communicating artery aneurysm requires careful exposure since the dome is frequently exposed in the interhemispheric fissure and attached to. the tuberculum sellae or the optic nerve. Sometimes it is necessary to detach the dome before clip application in order to expose the contralateral A1 and to prevent traction force on the neck induced by clip application. 


\section{Aneurysm of the internal carotid artery}

In surgery of the internal carotid artery aneurysm, wide opening of the Sylvian fissure and exposure of intracranial internal carotid artery as well as both proximal M1 and A1 are necessary. The posterior communicating artery with perforators and the pituitary stalk are better identified in space between the internal carotid artery and nearby optic nerve. Since the location and size of the posterior clinoid process are variable, it also should be initially defined prior to exposure of the aneurysm and application of a clip.

A special group of aneurysms include those that arise from the inferior (ventral) wall of the internal carotid artery and project inferiorly into the carotid and interpeduncular cistern. Of 593 intracranial aneurysms treated surgically, 10 aneurysms $(1.7 \%)$ were located on the inferior wall of the internal carotid artery. The aneurysms were large, had an ill-defined neck, fusiform in shape, often including more than half the circumsference of the internal carotid artery. They frequently showed sclerosis and were often partially thrombosed. Three cases had oculomotor nerve palsy. In the anterior-posterior view of the angiography they appeared as internal carotidophthalmic artery or bifurcation aneurysm, while in the lateral view they appeared to arise from the internal carotid-posterior communicating artery. In all cases a clip could be applied to the aneurysm only with considerable difficulty. Following exposure of the aneurysm through pterional craniotomy with low temporal muscle flap and wide opening of the Sylvian fissure, tedious dissection of the incorporated arteries and/or pituitary stalk was done. One or two angled aperture clip served to fashion an internal carotid artery from the aneurysm sac. Temporary clipping of the internal carotid artery in the neck was extremely helpful in obviating deliberate hypotension. In 2 cases, anterior clinoid process was ground to expose the neck of the aneurysm. Nine cases showed excellent recovery after clipping while one case died of occlusion of the internal carotid artery and infarction.

Combined extra- and intradural approach is indicated for an aneurysm located on $\mathrm{C} 2-3$ or paraclinoid area. In this procedure, the posterior one-third of the orbital roof, the roof of the optic canal and the anterior clinoid process were removed extradurally. An aneurysm which is arising from the dorsal surface of the internal carotid artery requires special attention to prevent premature rupture. It is usually safe if one first opens the Sylvian fissure and retracts the temporal lobe.

\section{Aneurysm of the middle cerebral artery}

Most of the aneurysm of the middle cerebral artery was exposed through a limited opening of the Sylvian fissure. Since the lesion is located in the extracerebral or the subarachnoid space, it was unnecessary to dissect already compromised cerebral tissue. Preoperative angiogram gives information regarding length of the M1 and direction of the aneurysm. A laterally directed aneurysm should be exposed carefully. Preoperative angiogram provides valuable information about the relationship of the lesion to the regional neurovascular and skeletal structures. The author found it useful to open the Sylvian fissure from the distal end and then expose the proximal middle cerebral trunk, which may be necessary for temporary occlusion in some cases. Since many of the aneurysms in this location have broad neck and multilobulation, careful bipolar coagulation is very helpful to shape a sac for complete clip application. One has to watch for perforators at the bifurcation of the middle cerebral artery.

\section{Aneurysm of the basilar artery}

Most of the basilar bifurcation aneurysms were treated by the pterional trans-Sylvian approach. Since this procedure provides adequate visualization of bilateral major branches at the basilar bifurcation, simultaneous management of multiple aneurysm located on the anterior circle of Willis and avoidance of temporal lobe retraction is possible. The subtemporal approach was used for posteriorly directed basilar bifurcation aneurysm. Lowlying basilar bifurcation aneurysms and basilar trunk aneurysms were treated subtemporal transtentorial approach.

\section{Reoperation of intracranial aneurysms which rebled after surgery}

Rupture of a treated aneurysm is one of the most formidable complications of aneurysm surgery. The author experienced six such cases among 818 aneurysms treated surgically in 756 patients. Two of the six cases were subjected to reoperation with good results. It is the author's suggestion that in order to avoid such a complication: surgery should be planned following a recent angiogram, as a ruptured aneurysm may grow in a few weeks; an intra- or postoperative angiography should be done if there was any unforeseen surgical finding; and microsurgical anatomy of the ruptured aneurysm must be fully appreciated.

\section{Unruptured Intracranial Aneurysm}

From a consecutive series of 777 aneurysms which the author has operated on during the last 13 years, 99 were unruptured aneurysms in 97 patients. "Unruptured aneurysms could be categorized into multiple aneursym, symptomatic aneurysm, and incidental aneurysm. Sixty- 
seven multiple aneurysms were treated in 65 patients. Sixty-five of these aneurysms were smaller than $10 \mathrm{~mm}$. Of the 23 symptomatic aneurysms, ptosis was the most frequent presenting symptom, followed by visual field defect. The size of the aneurysm was much larger in symptomatic aneurysms than in other unruptured aneurysms. Thirteen aneurysms were larger than $10 \mathrm{~mm}$, and five were giant aneurysms. Nine aneurysms were detected incidentally in nine patients while investigating such diseases as brain tumor, cerebral arteriovenous malformation, etc. Sizes of the aneurysms varied.

Two-thirds of the unruptured aneurysms were clipped and one-third were coagulated and/or reinforced. Giant aneurysms were treated by carotid ligation or balloon occlusion (Table 4). The morbidity and mortality of multiple aneurysms were due to ruptured aneurysms. The morbidity of two aneurysms in the symptomatic group was caused by worsening of the existing visual dysfunction in the patients with large aneurysms. One death in the symptomatic group was not directly related to the aneurysm surgery. There was no morbidity or mortality in the incidental group. If one considers the outcome of intact aneurysms only, there was no surgical mortality, and surgical morbidity was $2 \%$ (Table 5 ).

It is the author's conclusion that unruptured incidental or multiple aneurysms, especially if they are greater than $5 \mathrm{~mm}$ in diameter, should be treated surgically to prevent later rupture. The development of reliable noninvasive methods for aneurysm detection is of prime importance in the prevention of catastrophic rupture. Studies to understand the natural history of unruptured aneurysms and their optimal treatment are a logical corollary to noninvasive aneurysm detection.
Table 4 Operative Management of Unruptured Aneurysms.

\begin{tabular}{lcccr}
\hline \hline & Multiple & Symptomatic & Incidental & Total \\
\hline Clipping & 38 & 18 & 5 & 61 \\
Reinforcing & 29 & 3 & 4 & 33 \\
Carotid ligation & & 2 & & 3 \\
Balloon occlusion & & 23 & 9 & 99 \\
\hline Total & 67 & & &
\end{tabular}

Table 5 Operative Results of Unruptured Aneurysms.

\begin{tabular}{lcccc}
\hline & Good & Fair & Poor & Dead \\
\hline Multiple & 57 & 6 & 1 & 3 \\
Symptomatic & 20 & 2 & & 1 \\
Incidental & 9 & & & \\
\hline Total & 86 & 8 & 1 & 4 \\
\hline
\end{tabular}

\section{References}

1. Huh SK, Lee KC: The effect of the calcium antagonist nimodipine on cerebral cortical blood flow in the experimentally induced subarachnoid hemorrhage. J Korean Neurosurg Soc 16: 10911103, 1987 (in Korean)

2. Lee KS, Lee KC, Choi JU: Clinical trial of a calcium channel blocker in patients with aneurysmal subarachnoid hemorrhage prevention of delayed ischemic deficits. Yonsei Med J 28: 207220,1987

3. Lee KC: Postoperative intracerebral hemorrhage in aneurysm surgery. In: Intracranial Aneurysms and Arteriovenous Malformations, Sugita K, Shibuya M, eds, Nagoya University Coop Press, Nagoya, 1990, 335-341

4. Kim DS, Huh SK, Choi JU, Lee KC: Hydrocephalus in ruptured intracranial aneurysms. J Korean Neurosurg Soc 18: 917-925, 1989 (in Korean)

5. Lee KC: Surgical treatment of unruptured intracranial aneurysm. In: Intracranial Aneurysms, Kikuchi H, Fukushima T, Watanabe K, eds, Nishimura Co, Ltd, Niigata, 1986, 88-95 